Article

\title{
Fractional Hermite-Hadamard Integral Inequalities for a New Class of Convex Functions
}

\author{
Pshtiwan Othman Mohammed ${ }^{1, *(\mathbb{D})}$, Thabet Abdeljawad ${ }^{2,3,4, *} \mathbb{\infty}$, Shengda Zeng ${ }^{5}(\mathbb{D})$ and \\ Artion Kashuri ${ }^{6}$ iD \\ 1 Department of Mathematics, College of Education, University of Sulaimani, \\ Sulaimani 46001, Kurdistan Region, Iraq \\ 2 Department of Mathematics and General Sciences, Prince Sultan University, P.O. Box 66833, \\ Riyadh 11586, Saudi Arabia \\ 3 Department of Medical Research, China Medical University, Taichung 40402, Taiwan \\ 4 Department of Computer Science and Information Engineering, Asia University, Taichung 41354, Taiwan \\ 5 Jagiellonian University in Krakow, Faculty of Mathematics and Computer Science, ul. Lojasiewicza 6, \\ 30348 Krakow, Poland; shengda.zen@uj.edu.pl \\ 6 Department of Mathematics, Faculty of Technical Science, University Ismail Qemali, Vlora 9401, Albania; \\ artion.kashuri@univlora.edu.al \\ * Correspondence: pshtiwansangawi@gmail.com (P.O.M.); tabdeljawad@psu.edu.sa (T.A.)
}

Received: 15 August 2020; Accepted: 4 September 2020; Published: 9 September 2020

\begin{abstract}
Fractional integral inequality plays a significant role in pure and applied mathematics fields. It aims to develop and extend various mathematical methods. Therefore, nowadays we need to seek accurate fractional integral inequalities in obtaining the existence and uniqueness of the fractional methods. Besides, the convexity theory plays a concrete role in the field of fractional integral inequalities due to the behavior of its definition and properties. There is also a strong relationship between convexity and symmetric theories. So, whichever one we work on, we can then apply it to the other one due to the strong correlation produced between them, specifically in the last few decades. First, we recall the definition of $\varphi$-Riemann-Liouville fractional integral operators and the recently defined class of convex functions, namely the $\breve{\sigma}$-convex functions. Based on these, we will obtain few integral inequalities of Hermite-Hadamard's type for a $\breve{\sigma}$-convex function with respect to an increasing function involving the $\varphi$-Riemann-Liouville fractional integral operator. We can conclude that all derived inequalities in our study generalize numerous well-known inequalities involving both classical and Riemann-Liouville fractional integral inequalities. Finally, application to certain special functions are pointed out.
\end{abstract}

Keywords: $\varphi$-Riemann-Liouville fractional integral; convex functions; $\breve{\sigma}$-convex functions; Hermite-Hadamard inequality

\section{Introduction}

First of all, we recall the basic notations in convex analysis. A set $\chi \subset \mathbb{R}$ is said to be convex if

$$
\eta u+(1-\eta) v \in \chi
$$

for each $u, v \in \chi$ and $\eta \in[0,1]$. Based on a convex set $\chi$, we say that a function $Y: \chi \rightarrow \mathbb{R}$ is convex, if the inequality

$$
\mathrm{Y}(\eta u+(1-\eta) v) \leq \eta \mathrm{Y}(u)+(1-\eta) \mathrm{Y}(v), \quad \eta \in[0,1]
$$

holds for all $u, v \in \chi$. If $-\mathrm{Y}$ is convex, then we say that $\mathrm{Y}$ is concave. 
For the convex functions (1), there are many well-known integral inequalities in the literature; for example, Ostrowski integral inequalities [1], Simpson's integral inequalities [2], Hardy integral inequalities [3], Olsen integral inequalities [4], Gagliardo-Nirenberg integral inequalities [5], Hermite-Hadamard-Fejér integral inequalities [6] and q-Hermite-Hadamard integral inequalities [7]. Also, there is plenty of integral inequalities but the well-known one is the Hermite-Hadamard type integral inequality: the classical and fractional Hermite-Hadamard type integral inequalities $[8,9]$ are, respectively, given by:

$$
\mathrm{Y}\left(\frac{u+v}{2}\right) \leq \frac{1}{v-u} \int_{u}^{v} \mathrm{Y}(x) d x \leq \frac{\mathrm{Y}(u)+\mathrm{Y}(v)}{2}
$$

and

$$
\mathrm{Y}\left(\frac{u+v}{2}\right) \leq \frac{\Gamma(\wp+1)}{2(v-u)^{\wp}}\left[I_{u^{+}}^{\wp} \mathrm{Y}(v)+I_{v^{-}}^{\wp} \mathrm{Y}(u)\right] \leq \frac{\mathrm{Y}(u)+\mathrm{Y}(v)}{2}
$$

where $\mathrm{Y}: \chi \rightarrow \mathbb{R}$ is assumed to be a positive convex function on $\chi, \mathrm{Y} \in L^{1}(u, v)$ with $u<v$, and $I_{u^{+}}^{\wp}$ and $I_{v^{-}}^{\wp}$ stand for the left-sided and right-sided Riemann-Liouville fractional integrals of order $\wp>0$, respectively, and these are defined by [10]:

$$
\begin{aligned}
& I_{u^{+}}^{\wp} Y(x)=\frac{1}{\Gamma(\wp)} \int_{u}^{x}(x-\eta)^{\wp-1} Y(\eta) d \eta, \quad x>u ; \\
& I_{v^{-}}^{\wp} \mathrm{Y}(x)=\frac{1}{\Gamma(\wp)} \int_{x}^{v}(\eta-x)^{\wp-1} \mathrm{Y}(\eta) d \eta, \quad x<v .
\end{aligned}
$$

After introducing Hermite-Hadamard type inequalities (2) and (3), many classical and fractional integral inequalities have been established by a huge number of researcher; for more details one can see References [11-29].

Recently, in Reference [30], Wu et al. introduced a new class of convex sets and convex functions named $\breve{\sigma}$-convex sets and $\breve{\sigma}$-convex functions which are explained in the following definitions:

Definition 1. Let $\breve{\sigma} \subseteq \mathbb{R}$ be a strictly monotone and continuous function, and denote $\mathcal{M}_{[\breve{\sigma}, \eta]}(u, v):=\breve{\sigma}^{-1}(\eta \breve{\sigma}(u)+(1-\eta) \breve{\sigma}(v))$. We say that a set $\mathcal{Q} \subset \mathbb{R}$ is $\breve{\sigma}$-convex if

$$
\mathcal{M}_{[\breve{\sigma}, \eta]}(u, v) \in \mathcal{Q}, \quad \eta \in[0,1]
$$

for each $u, v \in \mathcal{Q}$.

Definition 2. A function $\mathrm{Y}: \mathcal{Q} \rightarrow \mathbb{R}$ is said to be $\breve{\sigma}$-convex if the inequality

$$
\mathrm{Y}\left(\mathcal{M}_{[\breve{r}, \eta]}(u, v)\right) \leq \eta \mathrm{Y}(u)+(1-\eta) \mathrm{Y}(v), \quad \eta \in[0,1]
$$

holds for each $u, v \in \mathcal{Q}$.

Remark 1. The function $\mathrm{Y}$ is called:

(i) strictly $\breve{\sigma}$-convex on $\mathcal{Q}$ if (6) is true as a strict inequality for all $\eta \in(0,1)$ and $u, v \in \mathcal{Q}$ with $u \neq v$.

(ii) $\breve{\sigma}$-concave on $\mathcal{Q}$, if $-\mathrm{Y}$ is $\breve{\sigma}$-convex on $\mathcal{Q}$.

(iii) strictly $\breve{\sigma}$-concave on $\mathcal{Q}$, if $-\mathrm{Y}$ is strictly $\breve{\sigma}$-convex on $\mathcal{Q}$.

Furthermore, in Reference [30], many inequalities of Hermite-Hadamard's type have been established using the notion of $\breve{\sigma}$-convexity. 
The aim of this article is to establish several inequalities of Hermite-Hadamard's type for $\breve{\sigma}$-convex functions via $\varphi$-Riemann-Liouville ( $\varphi-R L)$ fractional integrals, where the $\varphi-R L$ fractional integrals are defined as follows (see e.g., References [31,32]).

Definition 3. Let $(u, v) \subseteq \mathbb{R}, \varphi(x)$ be an increasing and positive function on $(u, v]$ and $\varphi^{\prime}(x)$ be continuous on $(u, v)$. Then, the left-sided and right-sided $\varphi-R L$ fractional integrals of a function $\mathrm{Y}$ with respect to the function $\varphi(x)$ on $[u, v]$ are respectively defined by [10,31-33]:

$$
\begin{aligned}
& I_{u^{+}}^{\wp: \varphi} Y(x)=\frac{1}{\Gamma(\wp)} \int_{u}^{x} \varphi^{\prime}(\eta)(\varphi(x)-\varphi(\eta))^{\wp-1} Y(\eta) d \eta ; \\
& I_{v^{-}}^{\wp: \varphi} Y(x)=\frac{1}{\Gamma(\wp)} \int_{x}^{v} \varphi^{\prime}(\eta)(\varphi(\eta)-\varphi(x))^{\wp-1} Y(\eta) d \eta, \quad \wp>0 .
\end{aligned}
$$

One can observe that if $\varphi$ is specialized by $\varphi(x)=x$, then $\varphi$-Riemann-Liouville fractional integral operators (7) reduce to the classical Riemann-Liouville fractional integral operators (4).

\section{Hermite-Hadamard's Type Inequalities for $\breve{\sigma}$-Convex Functions}

Our main results depend on the following lemmas:

Theorem 1. Assume that the function $\mathrm{Y}:[u, v] \subseteq \mathbb{R} \rightarrow \mathbb{R}$ is integrable $\breve{\sigma}$-convex and $\mathrm{Y} \in L^{1}(u, v)$ with $0 \leq u<v$. If the function $\breve{\sigma}$ is increasing and positive on $(u, v]$ and $\breve{\sigma}^{\prime}(x)$ is continuous on $(u, v)$. Then, we have for $\wp>0$ :

$$
\mathrm{Y}\left(\breve{\sigma}^{-1}\left(\frac{\breve{\sigma}(u)+\breve{\sigma}(v)}{2}\right)\right) \leq \frac{\Gamma(\wp+1)}{2(\breve{\sigma}(v)-\breve{\sigma}(u))^{\wp}}\left[I_{u^{+}}^{\wp: \breve{\sigma}} \mathrm{Y}(v)+I_{v^{-}}^{\wp: \breve{\sigma}} \mathrm{Y}(u)\right] \leq \frac{\mathrm{Y}(u)+\mathrm{Y}(v)}{2} .
$$

Proof. Since $\mathrm{Y}$ is a $\breve{\sigma}$-convex function, we have

$$
Y\left(\breve{\sigma}^{-1}\left(\frac{\breve{\sigma}(x)+\breve{\sigma}(y)}{2}\right)\right) \leq \frac{Y(x)+Y(y)}{2} .
$$

Substituting $x=\breve{\sigma}^{-1}(\eta \breve{\sigma}(u)+(1-\eta) \breve{\sigma}(v))$ and $y=\breve{\sigma}^{-1}((1-\eta) \breve{\sigma}(u)+\eta \breve{\sigma}(v))$ into (9), we get

$$
2 \mathrm{Y}\left(\breve{\sigma}^{-1}\left(\frac{\breve{\sigma}(u)+\breve{\sigma}(v)}{2}\right)\right) \leq \mathrm{Y}\left(\breve{\sigma}^{-1}(\eta \breve{\sigma}(u)+(1-\eta) \breve{\sigma}(v))\right)+\mathrm{Y}\left(\breve{\sigma}^{-1}((1-\eta) \breve{\sigma}(u)+\eta \breve{\sigma}(v))\right) .
$$

Multiplying both sides of (10) by $\eta^{\wp-1}$, then integrating the resulting inequality with respect to $\eta$ over $[0,1]$, we get

$$
\begin{aligned}
\frac{2}{\wp} Y\left(\breve{\sigma}^{-1}\left(\frac{\breve{\sigma}(u)+\breve{\sigma}(v)}{2}\right)\right) \leq \int_{0}^{1} \eta^{\wp-1} Y\left(\breve{\sigma}^{-1}(\eta \breve{\sigma}(u)\right. & +(1-\eta) \breve{\sigma}(v))) d \eta \\
& +\int_{0}^{1} \eta^{\wp-1} Y\left(\breve{\sigma}^{-1}((1-\eta) \breve{\sigma}(u)+\eta \breve{\sigma}(v))\right) d \eta .
\end{aligned}
$$

By changing the variables $z=\breve{\sigma}^{-1}(\eta \breve{\sigma}(u)+(1-\eta) \breve{\sigma}(v))$ and $w=\breve{\sigma}^{-1}((1-\eta) \breve{\sigma}(u)+\eta \breve{\sigma}(v))$, then the last inequality becomes

$$
\begin{aligned}
\mathrm{Y}\left(\breve{\sigma}^{-1}\left(\frac{\breve{\sigma}(u)+\breve{\sigma}(v)}{2}\right)\right) & \leq \frac{\wp}{2(\breve{\sigma}(v)-\breve{\sigma}(u))^{\wp}} \int_{u}^{v} \breve{\sigma}^{\prime}(z)(\breve{\sigma}(v)-\breve{\sigma}(z))^{\wp-1} Y(z) d z \\
& +\frac{\wp}{2(\breve{\sigma}(v)-\breve{\sigma}(u))^{\wp}} \int_{u}^{v} \breve{\sigma}^{\prime}(w)(\breve{\sigma}(w)-\breve{\sigma}(u))^{\wp-1} Y(w) d w \\
& =\frac{\Gamma(\wp+1)}{2(\breve{\sigma}(v)-\breve{\sigma}(u))^{\wp}}\left[I_{u^{+}}^{\wp: \varphi} Y(v)+I_{v^{-}}^{\wp \wp \varphi} Y(u)\right] .
\end{aligned}
$$


This completes the proof of our first inequality in (8).

In order to prove the second inequality in (8), we use the $\breve{\sigma}$-convexity of $Y$; that is

$$
\mathrm{Y}\left(\breve{\sigma}^{-1}(\eta \breve{\sigma}(u)+(1-\eta) \breve{\sigma}(v))\right) \leq \eta \mathrm{Y}(u)+(1-\eta) \mathrm{Y}(v),
$$

and

$$
\mathrm{Y}\left(\breve{\sigma}^{-1}((1-\eta) \breve{\sigma}(u)+\eta \breve{\sigma}(v))\right) \leq(1-\eta) \mathrm{Y}(u)+\eta \mathrm{Y}(v)
$$

By adding these two inequalities we get

$$
\begin{aligned}
\mathrm{Y}\left(\breve{\sigma}^{-1}(\eta \breve{\sigma}(u)+(1-\eta) \breve{\sigma}(v))\right)+\mathrm{Y}\left(\breve{\sigma}^{-1}((1-\eta) \breve{\sigma}(u)+\eta \breve{\sigma}(v))\right) \\
\leq \eta \mathrm{Y}(u)+(1-\eta) \mathrm{Y}(v)+(1-\eta) \mathrm{Y}(u)+\eta \mathrm{Y}(v) .
\end{aligned}
$$

Multiplying both sides of (11) by $\eta^{\wp-1}$ and then integrating with respect to $\eta$ over $[0,1]$, we can obtain

$$
\begin{aligned}
\int_{0}^{1} \eta^{\wp-1} \mathrm{Y}\left(\breve{\sigma}^{-1}(\eta \breve{\sigma}(u)\right. & +(1-\eta) \breve{\sigma}(v))) d \eta \\
& +\int_{0}^{1} \eta^{\wp-1} \mathrm{Y}\left(\breve{\sigma}^{-1}((1-\eta) \breve{\sigma}(u)+\eta \breve{\sigma}(v))\right) d \eta \leq(\mathrm{Y}(u)+\mathrm{Y}(v)) \int_{0}^{1} \eta^{\wp-1} d \eta
\end{aligned}
$$

hence,

$$
\frac{\Gamma(\wp+1)}{2(\breve{\sigma}(v)-\breve{\sigma}(u))^{\wp}}\left[I_{u^{+}}^{\wp: \breve{\sigma}} Y(v)+I_{v^{-}}^{\wp: \breve{\sigma}} Y(u)\right] \leq \frac{Y(u)+Y(v)}{2}
$$

This completes the proof of our Lemma 1.

Remark 2. Particularly, in Lemma 1, if we take

(i) $\breve{\sigma}(x)=x$, then inequality (8) reduces to inequality (3).

(ii) $\wp=1$, then we have

$$
\mathrm{Y}\left(\breve{\sigma}^{-1}\left(\frac{\breve{\sigma}(u)+\breve{\sigma}(v)}{2}\right)\right) \leq \frac{1}{\breve{\sigma}(v)-\breve{\sigma}(u)} \int_{u}^{v} \mathrm{Y}(x) \breve{\sigma}^{\prime}(x) d x \leq \frac{\mathrm{Y}(u)+\mathrm{Y}(v)}{2},
$$

which was already established in Reference [30].

(iii) $\breve{\sigma}(x)=x$ and $\wp=1$, then inequality (8) reduces to inequality (2).

Theorem 2. Assume that the function $\mathrm{Y}:[u, v] \subseteq \mathbb{R} \rightarrow \mathbb{R}$ is integrable $\breve{\sigma}$-convex and $\mathrm{Y} \in L^{1}(u, v)$ with $0 \leq u<v$. If the function $\breve{\sigma}$ is increasing and positive on $(u, v]$ and $\breve{\sigma}^{\prime}(x)$ is continuous on $(u, v)$. Then, we have for $\wp>0$ :

$$
\begin{array}{r}
Y\left(\breve{\sigma}^{-1}\left(\frac{\breve{\sigma}(u)+\breve{\sigma}(v)}{2}\right)\right) \leq \frac{\Gamma(\wp+1) 2^{\wp-1}}{(\breve{\sigma}(v)-\breve{\sigma}(u))^{\wp}}\left[I^{\wp: \breve{\sigma}}\left(\frac{\left.\breve{\sigma}^{-1}\left(\frac{\breve{\sigma}(u)+\breve{\sigma}(v)}{2}\right)\right)^{+}}{\left(v(v)+I^{\wp: \breve{\sigma}}\right.}+\left(\frac{\breve{\sigma}(u)+\breve{\sigma}(v)}{2}\right)\right)^{-}\right. \\
\leq \frac{Y(u)+Y(v)}{2}
\end{array}
$$


Proof. Again, since $\mathrm{Y}$ is a $\breve{\sigma}$-convex function, we can substituting $x=\breve{\sigma}^{-1}\left(\frac{\eta}{2} \breve{\sigma}(u)+\frac{2-\eta}{2} \breve{\sigma}(v)\right)$ and $y=\breve{\sigma}^{-1}\left(\frac{2-\eta}{2} \breve{\sigma}(u)+\frac{\eta}{2} \breve{\sigma}(v)\right)$ into (9) to get

$$
\begin{aligned}
2 Y\left(\breve{\sigma}^{-1}\left(\frac{\breve{\sigma}(u)+\breve{\sigma}(v)}{2}\right)\right) \leq \mathrm{Y}\left(\breve{\sigma}^{-1}\left(\frac{\eta}{2} \breve{\sigma}(u)+\frac{2-\eta}{2} \breve{\sigma}(v)\right)\right) & \\
& +\mathrm{Y}\left(\breve{\sigma}^{-1}\left(\frac{2-\eta}{2} \breve{\sigma}(u)+\frac{\eta}{2} \breve{\sigma}(v)\right)\right) .
\end{aligned}
$$

Multiplying both sides of (13) by $\eta^{\wp-1}$, then integrating the resulting inequality with respect to $\eta$ over $[0,1]$, we get

$$
\begin{aligned}
\frac{2}{\wp} Y\left(\breve{\sigma}^{-1}\left(\frac{\breve{\sigma}(u)+\breve{\sigma}(v)}{2}\right)\right) \leq \int_{0}^{1} \eta^{\wp-1} Y\left(\breve{\sigma}^{-1}(\right. & \left.\left.\frac{\eta}{2} \breve{\sigma}(u)+\frac{2-\eta}{2} \breve{\sigma}(v)\right)\right) d \eta \\
& +\int_{0}^{1} \eta^{\wp-1} Y\left(\breve{\sigma}^{-1}\left(\frac{2-\eta}{2} \breve{\sigma}(u)+\frac{\eta}{2} \breve{\sigma}(v)\right)\right) d \eta .
\end{aligned}
$$

By changing the variables $z=\breve{\sigma}^{-1}\left(\frac{\eta}{2} \breve{\sigma}(u)+\frac{2-\eta}{2} \breve{\sigma}(v)\right)$ and $w=\breve{\sigma}^{-1}\left(\frac{2-\eta}{2} \breve{\sigma}(u)+\frac{\eta}{2} \breve{\sigma}(v)\right)$, then the last inequality becomes

$$
\begin{aligned}
Y\left(\breve{\sigma}^{-1}\left(\frac{\breve{\sigma}(u)+\breve{\sigma}(v)}{2}\right)\right) & \leq \frac{\wp 2^{\wp-1}}{(\breve{\sigma}(v)-\breve{\sigma}(u))^{\wp}} \int_{\breve{\sigma}^{-1}\left(\frac{\breve{\sigma}(u)+\breve{\sigma}(v)}{2}\right)}^{v} \breve{\sigma}^{\prime}(z)(\breve{\sigma}(v)-\breve{\sigma}(z))^{\wp-1} Y(z) d z \\
& +\frac{\wp 2^{\wp-1}}{(\breve{\sigma}(v)-\breve{\sigma}(u))^{\wp}} \int_{u}^{\breve{\sigma}^{-1}\left(\frac{\breve{\sigma}(u)+\breve{\sigma}(v)}{2}\right)} \breve{\sigma}^{\prime}(w)(\breve{\sigma}(w)-\breve{\sigma}(u))^{\wp-1} \mathrm{Y}(w) d w \\
& =\frac{\Gamma(\wp+1) 2^{\wp-1}}{(\breve{\sigma}(v)-\breve{\sigma}(u))^{\wp}}\left[I^{\wp: \breve{\sigma}}\left(\breve{\sigma}^{-1}\left(\frac{\breve{\sigma}(u)+\breve{\sigma}(v)}{2}\right)\right)^{+} \mathrm{Y}(v)+I_{\left(\breve{\sigma}^{-1}\left(\frac{\breve{\sigma}(u)+\breve{\sigma}(v)}{2}\right)\right)^{-}}^{-} \mathrm{Y}(u)\right]
\end{aligned}
$$

This completes the proof of our first inequality in (12).

In order to obtain the second inequality in (12), we use the $\breve{\sigma}$-convexity of $Y$ as:

$$
\mathrm{Y}\left(\breve{\sigma}^{-1}\left(\frac{\eta}{2} \breve{\sigma}(u)+\frac{2-\eta}{2} \breve{\sigma}(v)\right)\right) \leq \frac{\eta}{2} \mathrm{Y}(u)+\frac{2-\eta}{2} \mathrm{Y}(v)
$$

and

$$
\mathrm{Y}\left(\breve{\sigma}^{-1}\left(\frac{2-\eta}{2} \breve{\sigma}(u)+\frac{\eta}{2} \breve{\sigma}(v)\right)\right) \leq \frac{2-\eta}{2} \mathrm{Y}(u)+\frac{\eta}{2} \mathrm{Y}(v)
$$

By adding these two inequalities we get

$$
\mathrm{Y}\left(\breve{\sigma}^{-1}\left(\frac{\eta}{2} \breve{\sigma}(u)+\frac{2-\eta}{2} \breve{\sigma}(v)\right)\right)+\mathrm{Y}\left(\breve{\sigma}^{-1}\left(\frac{2-\eta}{2} \breve{\sigma}(u)+\frac{\eta}{2} \breve{\sigma}(v)\right)\right) \leq \mathrm{Y}(u)+\mathrm{Y}(v) .
$$

Multiplying both sides of (14) by $\eta^{\wp-1}$ and then integrating with respect to $\eta$ over $[0,1]$, we get

$$
\begin{aligned}
\int_{0}^{1} \eta^{\wp-1} \mathrm{Y}\left(\breve{\sigma}^{-1}\left(\frac{\eta}{2} \breve{\sigma}(u)+\frac{2-\eta}{2} \breve{\sigma}(v)\right)\right) d \eta \\
\quad+\int_{0}^{1} \eta^{\wp-1} \mathrm{Y}\left(\breve{\sigma}^{-1}\left(\frac{2-\eta}{2} \breve{\sigma}(u)+\frac{\eta}{2} \breve{\sigma}(v)\right)\right) d \eta \leq(\mathrm{Y}(u)+\mathrm{Y}(v)) \int_{0}^{1} \eta^{\wp-1} d \eta
\end{aligned}
$$


namely,

$$
\frac{\Gamma(\wp+1) 2^{\wp-1}}{(\breve{\sigma}(v)-\breve{\sigma}(u))^{\wp}}\left[I^{\wp: \breve{\sigma}}\left(\breve{\sigma}^{-1}\left(\frac{\breve{\sigma}(u)+\breve{\sigma}(v)}{2}\right)\right)^{+} \mathrm{Y}(v)+I_{\left(\breve{\sigma}^{-1}\left(\frac{\breve{\sigma}(u)+\breve{\sigma}(v)}{2}\right)\right)^{-}}^{-\mathrm{Y}(u)}\right] \leq \frac{\mathrm{Y}(u)+\mathrm{Y}(v)}{2} .
$$

This completes the proof of our Lemma 2.

Remark 3. Especially, in Lemma 2, if we take

(i) $\breve{\sigma}(x)=x$, then inequality (12) reduces to the following inequality:

$$
\mathrm{Y}\left(\frac{u+v}{2}\right) \leq \frac{\Gamma(\wp+1) 2^{\wp-1}}{(v-u)^{\wp}}\left[I_{\left(\frac{u+v}{2}\right)^{+}}^{\wp} \mathrm{Y}(v)+I_{\left(\frac{u+v}{2}\right)^{-}}^{\wp} \mathrm{Y}(u)\right] \leq \frac{\mathrm{Y}(u)+\mathrm{Y}(v)}{2}
$$

which was already established in Reference [34].

(ii) $\wp=1$, then it holds

$$
\mathrm{Y}\left(\breve{\sigma}^{-1}\left(\frac{\breve{\sigma}(u)+\breve{\sigma}(v)}{2}\right)\right) \leq \frac{1}{\breve{\sigma}(v)-\breve{\sigma}(u)} \int_{u}^{v} \mathrm{Y}(x) \breve{\sigma}^{\prime}(x) d x \leq \frac{\mathrm{Y}(u)+\mathrm{Y}(v)}{2},
$$

which was already established in Reference [30].

(iii) $\breve{\sigma}(x)=x$ and $\wp=1$, then inequality (12) reduces to inequality (2).

\section{Further Consequences}

As consequences for the Lemmas 1 and 2, we can obtain the following theorems.

Theorem 3. Assume that $Y:[u, v] \subseteq \mathbb{R} \rightarrow \mathbb{R}$ is an $L^{1}$ integrable $\breve{\sigma}$-convex function and $Y^{\prime} \in L^{1}(u, v)$ with $0 \leq u<v$. If the function $\breve{\sigma}$ is increasing and positive on $(u, v]$ and $\breve{\sigma}^{\prime}(x)$ is continuous on $(u, v)$. Then, we have for $\wp>0$ :

$$
\begin{aligned}
& \frac{Y(u)+\mathrm{Y}(v)}{2}-\frac{\Gamma(\wp+1)}{2(\breve{\sigma}(v)-\breve{\sigma}(u))^{\wp}}\left[I_{u^{+}}^{\wp: \breve{\sigma}} \mathrm{Y}(v)+I_{v^{-}}^{\wp: \breve{\sigma}} \mathrm{Y}(u)\right] \\
& \quad=\frac{1}{2[\breve{\sigma}(v)-\breve{\sigma}(u)]^{\zeta}} \int_{u}^{v}\left((\breve{\sigma}(z)-\breve{\sigma}(u))^{\wp}-(\breve{\sigma}(v)-\breve{\sigma}(z))^{\wp}\right) \mathrm{Y}^{\prime}(z) d z .
\end{aligned}
$$

Proof. By Definition 3 and integrating by parts one can find

$$
\begin{aligned}
& I_{1}:=\frac{\Gamma(\wp+1)}{2(\breve{\sigma}(v)-\breve{\sigma}(u))^{\wp}} I_{u^{+}}^{\wp: \breve{\sigma}} \mathrm{Y}(v)=\frac{\wp}{2[\breve{\sigma}(v)-\breve{\sigma}(u)]^{\wp}} \int_{u}^{v} \breve{\sigma}^{\prime}(z)[\breve{\sigma}(v)-\breve{\sigma}(z)]^{\wp-1} \mathrm{Y}(z) d z \\
& =\frac{-1}{2[\breve{\sigma}(v)-\breve{\sigma}(u)]^{\wp}} \int_{u}^{v} \mathrm{Y}(z) d(\breve{\sigma}(v)-\breve{\sigma}(z))^{\wp} \\
& =\frac{1}{2[\breve{\sigma}(v)-\breve{\sigma}(u)]^{\wp}}\left[[\breve{\sigma}(v)-\breve{\sigma}(u)]^{\wp} \mathrm{Y}(u)+\int_{u}^{v}[\breve{\sigma}(v)-\breve{\sigma}(z)]^{\wp} \mathrm{Y}^{\prime}(z) d z\right] .
\end{aligned}
$$

Analogously, we get

$$
\begin{aligned}
I_{2}: & =\frac{\Gamma(\wp+1)}{2[\breve{\sigma}(v)-\breve{\sigma}(u)]^{\wp}} I_{v^{-}}^{\wp: \breve{\sigma}} Y(u)=\frac{\wp}{2[\breve{\sigma}(v)-\breve{\sigma}(u)]^{\wp}} \int_{u}^{v} \breve{\sigma}^{\prime}(z)[\breve{\sigma}(z)-\breve{\sigma}(u)]^{\wp-1} Y(z) d z \\
& =\frac{1}{2[\breve{\sigma}(v)-\breve{\sigma}(u)]^{\wp}}\left[[\breve{\sigma}(v)-\breve{\sigma}(u)]^{\wp} Y(v)-\int_{u}^{v}[\breve{\sigma}(z)-\breve{\sigma}(u)]^{\wp} Y^{\prime}(z) d z\right] .
\end{aligned}
$$


From the identities (16) and (17), it yields

$$
\frac{\mathrm{Y}(u)+\mathrm{Y}(v)}{2}-\left(I_{1}+I_{2}\right)=\frac{1}{2[\breve{\sigma}(v)-\breve{\sigma}(u)]^{\wp}} \int_{u}^{v}\left((\breve{\sigma}(z)-\breve{\sigma}(u))^{\wp}-(\breve{\sigma}(v)-\breve{\sigma}(z))^{\wp}\right) \mathrm{Y}^{\prime}(z) d z .
$$

This completes the proof of Theorem 3.

As a particular case of Theorem 3 , if $\breve{\sigma}$ is specialized by $\breve{\sigma}(z)=z$, then we have the following corollary, which has been studied by Sarikaya et al. in Reference [9].

Corollary 1. Under the same assumptions of Theorem 3 , if $\breve{\sigma}(z)=z$, then we have

$$
\frac{Y(u)+Y(v)}{2}-\frac{\Gamma(\wp+1)}{2(v-u)^{\wp}}\left[I_{u^{+}}^{\wp} Y(v)+I_{v^{-}}^{\wp} Y(u)\right]=\frac{1}{2(v-u)^{\wp}} \int_{u}^{v}\left[(z-u)^{\wp}-(v-z)^{\wp}\right] Y^{\prime}(z) d z .
$$

Moreover, if $\left|\mathrm{Y}^{\prime}\right|$ is convex on $[u, v]$, then we have

$$
\left|\frac{\mathrm{Y}(u)+\mathrm{Y}(v)}{2}-\frac{\Gamma(\wp+1)}{2(v-u)^{\wp}}\left[I_{u^{+}}^{\wp} \mathrm{Y}(v)+I_{v^{-}}^{\wp} \mathrm{Y}(u)\right]\right| \leq \frac{v-u}{2(\wp+1)}\left(1-\frac{1}{2^{\wp}}\right)\left[\left|\mathrm{Y}^{\prime}(u)\right|+\left|\mathrm{Y}^{\prime}(v)\right|\right] .
$$

Proof. By putting $\breve{\sigma}(z)=z$ into Theorem 3 , we directly obtain the desired equality (18). To prove the inequality (19), we change the variable $z=(1-\eta) u+\eta v$ in (18), we have

$$
\frac{\mathrm{Y}(u)+\mathrm{Y}(v)}{2}-\frac{\Gamma(\wp+1)}{2(v-u)^{\wp}}\left[I_{u^{+}}^{\wp} \mathrm{Y}(v)+I_{v^{-}}^{\wp} \mathrm{Y}(u)\right]=\frac{v-u}{2} \int_{0}^{1}\left[(1-\eta)^{\wp}-\eta^{\wp}\right] \mathrm{Y}^{\prime}((1-\eta) u+\eta v) d \eta .
$$

Then, we obtain (19) as in the proof of [9, Theorem 3].

Theorem 4. Assume that $\mathrm{Y}:[u, v] \subseteq \mathbb{R} \rightarrow \mathbb{R}$ is an $L^{1}$ integrable $\breve{\sigma}$-convex function and $\mathrm{Y}^{\prime} \in L^{1}(u, v)$ with $0 \leq u<v$. If the function $\breve{\sigma}$ is increasing and positive on $(u, v]$ and $\breve{\sigma}^{\prime}(x)$ is continuous on $(u, v)$. Then, we have

$$
\begin{aligned}
& \frac{\Gamma(\wp+1) 2^{\wp-1}}{(\breve{\sigma}(v)-\breve{\sigma}(u))^{\wp}}\left[I^{\wp: \breve{\sigma}}\left(\breve{\sigma}^{-1}\left(\frac{\breve{\sigma}(u)+\breve{\sigma}(v)}{2}\right)\right)^{+} \mathrm{Y}(v)+I^{\wp: \breve{\sigma}}\left(\breve{\sigma}^{-1}\left(\frac{\breve{\sigma}(u)+\breve{\sigma}(v)}{2}\right)\right)^{-} \mathrm{Y}(u)\right]-\mathrm{Y}\left(\breve{\sigma}^{-1}\left(\frac{\breve{\sigma}(u)+\breve{\sigma}(v)}{2}\right)\right) \\
& =\frac{2^{\wp-1}}{(\breve{\sigma}(v)-\breve{\sigma}(u))^{\wp}}\left(\int_{\breve{\sigma}^{-1}\left(\frac{\breve{\sigma}(u)+\check{\sigma}(v)}{2}\right)}^{v}(\breve{\sigma}(v)-\breve{\sigma}(z))^{\wp} Y^{\prime}(z) d z\right. \\
& \left.-\int_{u}^{\breve{\sigma}^{-1}\left(\frac{\breve{\sigma}(u)+\breve{\sigma}(v)}{2}\right)}(\breve{\sigma}(z)-\breve{\sigma}(u))^{\wp} Y^{\prime}(z) d z\right)
\end{aligned}
$$

for $\wp>0$. 
Proof. By Definition 3 and integrating by parts one can find

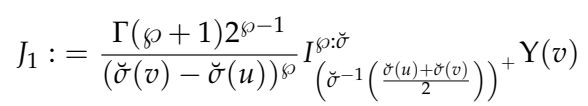

$$
\begin{aligned}
& =\frac{\wp 2^{\wp-1}}{(\breve{\sigma}(v)-\breve{\sigma}(u))^{\wp}} \int_{\breve{\sigma}^{-1}\left(\frac{\breve{\sigma}(u)+\check{(}(v)}{2}\right)}^{v} \breve{\sigma}^{\prime}(z)(\breve{\sigma}(v)-\breve{\sigma}(z))^{\wp-1} Y(z) d z
\end{aligned}
$$

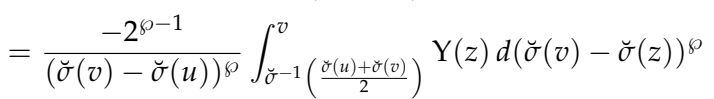

$$
\begin{aligned}
& =\frac{2^{\wp-1}}{(\breve{\sigma}(v)-\breve{\sigma}(u))^{\wp}}\left[\left(\frac{\breve{\sigma}(v)-\breve{\sigma}(u)}{2}\right)^{\wp} \mathrm{Y}\left(\breve{\sigma}^{-1}\left(\frac{\breve{\sigma}(u)+\breve{\sigma}(v)}{2}\right)\right)+\int_{\breve{\sigma}^{-1}\left(\frac{\breve{\sigma}(u)+\breve{\sigma}(v)}{2}\right)}^{v}(\breve{\sigma}(v)-\breve{\sigma}(z))^{\wp} \mathrm{Y}^{\prime}(z) d z\right] \\
& =\frac{1}{2} \mathrm{Y}\left(\breve{\sigma}^{-1}\left(\frac{\breve{\sigma}(u)+\breve{\sigma}(v)}{2}\right)\right)+\frac{2^{\wp-1}}{(\breve{\sigma}(v)-\breve{\sigma}(u))^{\wp}} \int_{\breve{\sigma}^{-1}}^{v}\left(\frac{\breve{\sigma}(u)+\check{\sigma}(v)}{2}\right)(\breve{\sigma}(v)-\breve{\sigma}(z))^{\wp} \mathrm{Y}^{\prime}(z) d z .
\end{aligned}
$$

Similarly, we have

$$
\begin{aligned}
J_{2}: & =\frac{\Gamma(\wp+1) 2^{\wp-1}}{(\breve{\sigma}(v)-\breve{\sigma}(u))^{\wp}} I^{\wp: \breve{\sigma}}\left(\breve{\sigma}^{-1}\left(\frac{\breve{\sigma}(u)+\breve{\sigma}(v)}{2}\right)\right)^{-} Y(u) \\
& =\frac{\wp 2^{\wp-1}}{(\breve{\sigma}(v)-\breve{\sigma}(u))^{\wp}} \int_{u}^{\breve{\sigma}^{-1}\left(\frac{\breve{\sigma}(u)+\breve{\sigma}(v)}{2}\right)} \breve{\sigma}^{\prime}(z)(\breve{\sigma}(z)-\breve{\sigma}(u))^{\wp-1} Y(z) d z \\
& =\frac{1}{2} Y\left(\breve{\sigma}^{-1}\left(\frac{\breve{\sigma}(u)+\breve{\sigma}(v)}{2}\right)\right)-\frac{2^{\wp-1}}{(\breve{\sigma}(v)-\breve{\sigma}(u))^{\wp}} \int_{u}^{\breve{\sigma}^{-1}\left(\frac{\breve{\sigma}(u)+\breve{\sigma}(v)}{2}\right)}(\breve{\sigma}(z)-\breve{\sigma}(u))^{\wp} Y^{\prime}(z) d z .
\end{aligned}
$$

From the identities (21) and (22), one has

$$
\begin{aligned}
& J_{1}+J_{2}-Y\left(\breve{\sigma}^{-1}\left(\frac{\breve{\sigma}(u)+\breve{\sigma}(v)}{2}\right)\right) \\
& =\frac{2^{\wp-1}}{(\breve{\sigma}(v)-\breve{\sigma}(u))^{\wp}}\left(\int_{\breve{\sigma}^{-1}\left(\frac{\breve{\sigma}(u)+\breve{\sigma}(v)}{2}\right)}^{v}(\breve{\sigma}(v)-\breve{\sigma}(z))^{\wp} Y^{\prime}(z) d z-\int_{u}^{\breve{\sigma}^{-1}\left(\frac{\breve{\sigma}(u)+\breve{\sigma}(v)}{2}\right)}(\breve{\sigma}(z)-\breve{\sigma}(u))^{\wp} Y^{\prime}(z) d z\right) .
\end{aligned}
$$

This ends the proof of our Theorem 4 .

Additionally, when $\breve{\sigma}(z)=z$, then our result Theorem 4 becomes to the following corollary, which has been already explored by Sarikaya et al. in [34].

Corollary 2. Particularly, if we take $\breve{\sigma}(z)=z$ in Theorem 4 , we get

$$
\begin{aligned}
\frac{\Gamma(\wp+1) 2^{\wp-1}}{(v-u)^{\wp}}\left[I_{\left(\frac{u+v}{2}\right)^{+}}^{\wp} \mathrm{Y}(v)+\right. & \left.I_{\left(\frac{u+v}{2}\right)^{-}}^{\wp} \mathrm{Y}(u)\right]-\mathrm{Y}\left(\frac{u+v}{2}\right) \\
& =\frac{2^{\wp-1}}{(v-u)^{\wp}}\left(\int_{\frac{u+v}{2}}^{v}(v-z)^{\wp} \mathrm{Y}^{\prime}(z) d z-\int_{u}^{\frac{u+v}{2}}(z-u)^{\wp} \mathrm{Y}^{\prime}(z) d z\right) .
\end{aligned}
$$

Moreover, if $\left|Y^{\prime}\right|^{q}, q \geq 1$ is convex on $[u, v]$, we have

$$
\begin{aligned}
\left|\frac{\Gamma(\wp+1) 2^{\wp-1}}{(v-u)^{\wp}}\left[I_{\left(\frac{u+v}{2}\right)^{+}}^{\wp} \mathrm{Y}(v)+I_{\left(\frac{u+v}{2}\right)^{-}}^{\wp} \mathrm{Y}(u)\right]-\mathrm{Y}\left(\frac{u+v}{2}\right)\right| & \leq \frac{v-u}{4(\wp+1)}\left(\frac{1}{2(\wp+2)}\right)^{\frac{1}{q}}\left[\left((\wp+1)\left|\mathrm{Y}^{\prime}(u)\right|^{q}+(\wp+3)\left|\mathrm{Y}^{\prime}(v)\right|^{q}\right)^{\frac{1}{q}}\right. \\
& \left.+\left((\wp+3)\left|\mathrm{Y}^{\prime}(u)\right|^{q}+(\wp+1)\left|\mathrm{Y}^{\prime}(v)\right|^{q}\right)^{\frac{1}{q}}\right] .
\end{aligned}
$$


Proof. By putting $\breve{\sigma}(z)=z$ into Theorem 4 , we directly obtain the desired equality (23). To prove the inequality (24), we use the same proof technique of Corollary 1 with the convexity of $\left|Y^{\prime}\right|^{q}$. Thus, the proof of Corollary 2 is completed.

\section{Applications}

\subsection{The Modified Bessel Functions}

Consider the function $\mathcal{W}_{\zeta}: \mathbb{R} \rightarrow[1, \infty)$, defined by:

$$
\mathcal{W}_{\zeta}(z)=2^{\varsigma} \Gamma(\varsigma+1) z^{-\varsigma} S_{\zeta}(z), \quad z \in \mathbb{R}, \varsigma>-1 .
$$

Then, the modified Bessel function of the first $S_{\zeta}$ and the second kind $\mathcal{Y}_{\zeta}$ are defined as follows [35]:

$$
\begin{aligned}
& S_{\zeta}(z)=\sum_{n=0}^{\infty} \frac{\left(\frac{z}{2}\right)^{\varsigma+2 n}}{n ! \Gamma(\varsigma+n+1)} ; \\
& \mathcal{Y}_{\zeta}(z)=\frac{\pi}{2} \frac{S_{-\varsigma}(z)+S_{\zeta}(z)}{\sin (\varsigma \pi)} .
\end{aligned}
$$

respectively.

Proposition 1. Assume that $u, v \in \mathbb{R}$ with $0<u<v$. Then, we have for each $\varsigma>-1$ :

$$
\left|\frac{\left(\mathcal{W}_{\varsigma} \circ \breve{\sigma}\right)(v)-\left(\mathcal{W}_{\varsigma} \circ \breve{\sigma}\right)(u)}{\breve{\sigma}(v)-\breve{\sigma}(u)}\right| \leq \frac{\breve{\sigma}(u)\left(\mathcal{W}_{\zeta+1} \circ \breve{\sigma}\right)(u)+\breve{\sigma}(v)\left(\mathcal{W}_{\zeta+1} \circ \breve{\sigma}\right)(v)}{4(\varsigma+1)}
$$

where $\breve{\sigma}$ as before.

Proof. Following Reference [35], we have

$$
\mathcal{W}_{\varsigma}^{\prime}(z)=\frac{z}{2(\varsigma+1)} \mathcal{W}_{\varsigma+1}(z)
$$

One can observe that the function $\mathcal{W}_{\zeta}^{\prime}(z)$ is convex on $(0, \infty)$ for $\varsigma>-1$. So, the function $Y(z):=\left(\mathcal{W}_{\varsigma}^{\prime} \circ \breve{\sigma}\right)(z)$ is $\breve{\sigma}$-convex on the same interval. Using (26) and Lemma 1 with $\wp=1$, we obtain the desired inequality (25) immediately.

Proposition 2. Assume that $u, v \in \mathbb{R}$ with $0<u<v$. Then, we have for each $\varsigma>-1$ :

$$
\left|\frac{\breve{\sigma}^{\zeta}(u)\left(\mathcal{Y}_{\varsigma} \circ \breve{\sigma}\right)(v)-\breve{\sigma} \breve{\sigma}^{\varsigma}(v)\left(\mathcal{Y}_{\varsigma} \circ \breve{\sigma}\right)(u)}{\breve{\sigma}(v)-\breve{\sigma}(u)}\right| \leq \frac{\breve{\sigma}^{\varsigma}(v)\left(\mathcal{Y}_{\varsigma+1} \circ \breve{\sigma}\right)(u)+\breve{\sigma}^{\varsigma}(u)\left(\mathcal{Y}_{\varsigma+1} \circ \breve{\sigma}\right)(v)}{2},
$$

where $\breve{\sigma}$ as before.

Proof. Let $f_{\varsigma}(z):=\frac{\mathcal{Y}_{\varsigma}(z)}{z^{\zeta}}$, where $\varsigma \in \mathbb{R}$. Consider the integral formula [35]:

$$
\mathcal{Y}_{\varsigma}(z)=\int_{0}^{\infty} e^{-z \cosh (\eta)} \cosh (\varsigma \eta) d \eta, \quad z>0 .
$$

One can note that the function $z \mapsto \mathcal{Y}_{\varsigma}(z)$ is completely monotonic on $(0, \infty)$ for any $\varsigma \in \mathbb{R}$. Then, we conclude that $z \mapsto f_{\zeta}(z)$ is strictly complete monotonic on $(0, \infty)$ for each $\varsigma>-1$ since the product of two strictly completely monotonic functions is strictly completely monotonic as well. Therefore, the function $-f_{\varsigma}^{\prime}(z)=-\left(\frac{\mathcal{Y}_{\zeta}(z)}{z^{\zeta}}\right)^{\prime}=-\frac{\mathcal{Y}_{\zeta+1}(z)}{z^{\zeta}}$ is strictly completely monotonic on $(0, \infty)$ for any $\varsigma>-1$ and thus $f_{\varsigma}$ is convex. 
Now, setting $Y(z):=-\left(f_{\varsigma}^{\prime} \circ \breve{\sigma}\right)(z)$, then one can conclude that the function $Y(z)$ is $\breve{\sigma}$-convex. Consequently, by applying Lemma 1 with above $Y(z)$ and $\wp=1$, we can obtain the desired inequality (27) immediately for $\varsigma>-1$.

\subsection{Special Means}

We consider the special means of positive numbers $u \neq v$ :

- The arithmetic mean:

$$
\mathbf{A}(u, v)=\frac{u+v}{2}
$$

- The generalized logarithmic mean:

$$
\mathbf{L}_{\zeta}(u, v)=\left[\frac{v^{\varsigma+1}-u^{\varsigma+1}}{(\varsigma+1)(v-u)}\right]^{\frac{1}{\varsigma}}, \quad \varsigma \in \mathbb{R} \backslash\{-1,0\} .
$$

Proposition 3. Let $0<u<v$, where $u, v \in \mathbb{R}$. Then, we have

$$
\ln ^{2}\left[A\left(e^{u}, e^{v}\right)\right] \leq v^{2} e^{v}-u^{2} e^{u}-4 A\left((1-u) e^{u},(v-1) e^{v}\right) \leq A\left(u^{2}, v^{2}\right) .
$$

Proof. Taking $\mathrm{Y}(z)=z^{2}, \breve{\sigma}(z)=e^{z}$ and $\wp=1$ in Remark 2 part (ii), the double inequality (28) is obtained.

Proposition 4. Let $0<u<v$, where $u, v \in \mathbb{R}$. Then for $\varsigma>0$, we get

$$
A^{\frac{2}{\varsigma}}\left(u^{\varsigma}, v^{\varsigma}\right) \leq \frac{\varsigma(v-u)}{v^{\varsigma}-u^{\varsigma}} L_{\varsigma+1}^{\varsigma+1}(u, v) \leq A\left(u^{2}, v^{2}\right)
$$

Proof. Choosing $Y(z)=z^{2}, \breve{\sigma}(z)=z^{\varsigma}$ and $\wp=1$ in Remark 2 part (ii), the double inequality (29) is captured.

\section{Conclusions}

In the study, we have considered a new class of convex functions and the definition of $\varphi-\mathrm{RL}$ fractional integral operators. In our present investigation, we have established new fractional Hermite-Hadamard's integral inequalities associated to increasing functions. The results obtained here are very useful in obtaining other type of inequalities. Also, these results are very generic and can be specified to give further potentially useful and interesting integral inequalities involving other type of fractional integral operators.

Author Contributions: Conceptualization, P.O.M. and T.A.; methodology, S.Z.; software, P.O.M.; validation, P.O.M., S.Z. and A.K.; formal analysis, P.O.M.; investigation, P.O.M.; resources, T.A.; data curation, A.K.; writing—original draft preparation, P.O.M.; writing—review and editing, S.Z.; visualization, A.K.; supervision, T.A.; project administration, S.Z.; funding acquisition, T.A. All authors have read and agreed to the published version of the manuscript.

Funding: This research received no external funding.

Acknowledgments: The authors express their special thanks to the Associate Editor and the referees.

Conflicts of Interest: The authors declare no conflict of interest.

\section{References}

1. Gavrea, B.; Gavrea, I. On some Ostrowski type inequalities. Gen. Math. 2010, 18, 33-44.

2. Vivas-Cortez, M.; Abdeljawad, T.; Mohammed, P.O.; Rangel-Oliveros, Y. Simpson's Integral Inequalities for Twice Differentiable Convex Functions. Math. Probl. Eng. 2020, 2020, 1936461. [CrossRef] 
3. Kaijser, S.; Nikolova, L.; Persson, L.-E.; Wedestig, A. Hardy type inequalities via convexity. Math. Inequal. Appl. 2005, 8, 403-417. [CrossRef]

4. Gunawan, H.; Eridani. Fractional integrals and generalized Olsen inequalities. Kyungpook Math. J. 2009, 49, 31-39. [CrossRef]

5. Sawano, Y.; Wadade, H. On the Gagliardo-Nirenberg type inequality in the critical Sobolev-Morrey space. J. Fourier Anal. Appl. 2013, 19, 20-47. [CrossRef]

6. Kunt, M.; Iscan, I. Hermite-Hadamard-Fejér type inequalities for p-convex functions. Arab J. Math. Sci. 2017, 23, 215-230. [CrossRef]

7. Alp, N.; Sarkkaya, M.Z.; Kunt, M.; Iscan, I. q-Hermite Hadamard inequalities and quantum estimates for midpoint type inequalities via convex and quasi-convex functions. J. King Saud Univ. Sci. 2018, 30, 193-203. [CrossRef]

8. Hadamard, J. Étude sur les propriétés des fonctions entières en particulier d'une fonction considérée par Riemann. J. Math. Pures Appl. 1893, 58, 171-215.

9. Sarikaya, M.Z.; Set, E.; Yaldiz, H.; Başak, N. Hermite-Hadamard's inequalities for fractional integrals and related fractional inequalities. Math. Comput. Model. 2013, 57, 2403-2407. [CrossRef]

10. Kilbas, A.A.; Srivastava, H.M.; Trujillo, J.J. Theory and Applications of Fractional Differential Equations; North-Holland Mathematics Studies; Elsevier Sci. B.V.: Amsterdam, The Netherlands, 2006; Volume 204.

11. Dragomir, S.S.; Pearce, C.E.M. Selected Topics on Hermite-Hadamard Inequalities and Applications; RGMIA Monographs; Victoria University: Footscray, Australia, 2000.

12. Ozdemir, M.E.; Avci, M.; Kavurmaci, H. Hermite-Hadamard-type inequalities via $(\alpha, m)$-convexity. Comput. Math. Appl. 2011, 61, 2614-2620. [CrossRef]

13. Fernandez, A.; Mohammed, P. Hermite-Hadamard inequalities in fractional calculus defined using Mittag-Leffler kernels. Math. Meth. Appl. Sci. 2020, 1-18. [CrossRef]

14. Mohammed, P.O. Some new Hermite-Hadamard type inequalities for MT-convex functions on differentiable coordinates. J. King Saud Univ. Sci. 2018, 30, 258-262. [CrossRef]

15. Abdeljawad, T.; Mohammed, P.O.; Kashuri, A. New Modified Conformable Fractional Integral Inequalities of Hermite-Hadamard Type with Applications. J. Funct. Space 2020, 2020, 4352357. [CrossRef]

16. Mohammed, P.O.; Sarikaya, M.Z.; Baleanu, D. On the Generalized Hermite-Hadamard Inequalities via the Tempered Fractional Integrals. Symmetry 2020, 12, 595. [CrossRef]

17. Baleanu, D.; Mohammed, P.O.; Zeng, S. Inequalities of trapezoidal type involving generalized fractional integrals. Alex. Eng. J. 2020. [CrossRef]

18. Baleanu, D.; Mohammed, P.O.; Vivas-Cortez, M.; Rangel-Oliveros, Y. Some modifications in conformable fractional integral inequalities. Adv. Differ. Equ. 2020, 2020, 374. [CrossRef]

19. Mohammed, P.O.; Sarikaya, M.Z. Hermite-Hadamard type inequalities for F-convex function involving fractional integrals. J. Inequal. Appl. 2018, 2018, 359. [CrossRef]

20. Han, J.; Mohammed, P.O.; Zeng, H. Generalized fractional integral inequalities of Hermite-Hadamard-type for a convex function. Open Math. 2020, 18, 794-806. [CrossRef]

21. Mohammed, P.O.; Sarikaya, M.Z. On generalized fractional integral inequalities for twice differentiable convex functions. J. Comput. Appl. Math. 2020, 372, 112740. [CrossRef]

22. Mohammed, P.O. On New Trapezoid Type Inequalities for $h$-convex Functions via Generalized Fractional Integral. Turk. J. Anal. Number. Theory 2018, 6, 125-128. [CrossRef]

23. Mohammed, P.O. Inequalities of Type Hermite-Hadamard for Fractional Integrals via Differentiable Convex Functions. Turk. J. Anal. Number. Theory 2016, 4, 135-139.

24. Qi, F.; Mohammed, P.O.; Yao, J.C.; Yao, Y.H. Generalized fractional integral inequalities of Hermite-Hadamard type for $(\alpha, m)$-convex functions. J. Inequal. Appl. 2019, 2019, 135. [CrossRef]

25. Mohammed, P.O. Fractional integral inequalities of Hermite-Hadamard type for convex functions with respect to a monotone function. arXiv 2020, arXiv:2005.00588.

26. Mohammed, P.O. Hermite-Hadamard inequalities for Riemann-Liouville fractional integrals of a convex function with respect to a monotone function. Math. Meth. Appl. Sci. 2019, 1-11. [CrossRef]

27. Mohammed, P.O.; Abdeljawad, T. Modification of certain fractional integral inequalities for convex functions. Adv. Differ. Equ. 2020, 2020, 69. [CrossRef]

28. Mohammed, P.O.; Abdeljawad, T. Integral inequalities for a fractional operator of a function with respect to another function with nonsingular kernel. Adv. Differ. Equ. 2020, 2020, 363. [CrossRef] 
29. Mohammed, P.O.; Brevik, I. A New Version of the Hermite-Hadamard Inequality for Riemann-Liouville Fractional Integrals. Symmetry 2020, 12, 610. [CrossRef]

30. Wu, S.; Awan, M.U.; Noor, M.A.; Noor, K.I.; Iftikhar, S. On a new class of convex functions and integral inequalities. J. Inequal. Appl. 2019, 2019, 131. [CrossRef]

31. Osler, T.J. The Fractional Derivative of a Composite Function. SIAM J. Math. Anal. 1970, 1, $288-293$. [CrossRef]

32. Almeida, R. A Caputo fractional derivative of a function with respect to another function. Commun. Nonlinear Sci. Numer. Simul. 2017, 44, 460-481. [CrossRef]

33. Sousa, J.V.C.; Oliveira, E.C. On the $\Psi$-Hilfer fractional derivative. Commun. Nonlinear Sci. Numer. Simul. 2018, 60, 72-91. [CrossRef]

34. Sarikaya, M.Z.; Yildirim, H. On Hermite-Hadamard type inequalities for Riemann-Liouville fractional integrals. Miskolc Math. Notes 2017, 17, 1049-1059. [CrossRef]

35. Watson, G.N. A Treatise on the Theory of Bessel Functions; Cambridge University Press: Cambridge, UK, 1944.

(C) 2020 by the authors. Licensee MDPI, Basel, Switzerland. This article is an open access article distributed under the terms and conditions of the Creative Commons Attribution (CC BY) license (http:/ / creativecommons.org/licenses/by/4.0/). 